through genital hygiene practises or other practises (e.g.female genital mutilation or masturbation) should be explored to determine the possibility of HPV acquisition prior to first sex, which may have implications for vaccination programmes.

\section{P3.057 CLINICAL FOLLOW-UP OF WOMEN WITH GENITAL HUMAN PAPILLOMAVIRUS INFECTION TREATED AT A REFERENCE HOSPITAL IN BRAZIL}

doi:10.1136/sextrans-2013-051184.0517

1J Gaspar, 'E Gir, 'R K Reis, ${ }^{2}$ S M Quintana, ''S R M S Canini. 'Nursing School of Ribeirão Preto, University of São Paulo, Ribeirão Preto, Brazil; '²Faculty Of Medicine of Ribeirão Preto, University of São Paulo, Ribeirão Preto, Brazil

Background The viral types of HPV are classified as low or high risk oncogenic. The low risk is associated with benign genital tract infections such as genital warts or flat intraepithelial lesions of lowgrade (LSIL). Already the high risk have a high correlation with intraepithelial lesion high-grade (HSIL) and carcinoma of the cervix, vulva, anus and, more rarely, the penis. Cancer of the cervix is the second most common type of cancer among women, with approximately 500,000 new cases per year worldwide. Therefore, the aim of this study was to evaluate the clinical follow-up of women with HSIL caused by HPV, considering the attendance and the number of appointments after undergoing surgery for high frequency (CAF).

Methods Transversal retrospective study with a quantitative approach, conducted in the Department of Infectious Diseases in Obstetrics and Gynecology (SEMIGO) of the Hospital of the Faculty of Medicine of Ribeirão Preto, University of São Paulo, Brazil. The study population was composed of 169 women diagnosed with HSIL caused by HPV, which were submitted to CAF for at least 24 months. We analysed attendance in six of those women returns by pre-established protocol of care service study for the period of 24 months after completion of CAF.

Results Regarding the clinical follow-up, 108 (63.9\%) women attended the first return after LEEP, $116(68.6 \%)$ returned the second, $72(42.6 \%)$ to the third return, $74(43.8 \%)$ to the fourth return, $67(39.6 \%)$ the fifth feedback and $67(39.6 \%)$ to the sixth return.

Conclusion Considering the decline in attendance at scheduled appointments over the 24 months, it is necessary to implement health programmes aimed at greater control of clinical follow-up actions promoting character education, developed with the participation of a multidisciplinary team.

\section{P3.058 UTILIZATION OF MUNICIPAL STD CLINIC SERVICES AMONG THE INSURED, SAN FRANCISCO 2011-2: IMPLICATIONS FOR HEALTH CARE REFORM IN THE UNITED STATES}

doi:10.1136/sextrans-2013-051184.0518

S C Stephens, S S Philip, S E Cohen, K T Bernstein. San Francisco Department of Public Health, San Francisco, CA, United States

Limited data exist on the number of insured patients who receive care at publically funded STD clinics, despite having access to a primary care provider. In this analysis, we compare patients with and without health insurance who sought services at City Clinic, the San Francisco municipal STD clinic.

We analysed San Francisco City Clinic visits between August 1, 2011 and August 31, 2012. Insurance was self-reported at registration and included both private and public insurance. Variables from the clinic electronic medical record were examined and included basic demographic and risk behaviour questions, as well as positivity among asymptomatic patients tested for vaginal, urethral, rectal, pharyngeal and/or rectal chlamydial and gonoccocal infection. We compared the characteristics of insured and uninsured patients using chi-square statistics.
There were 18,232 patient visits in this analysis, of which 6,305 (35\%) were categorised as insured and 11,927 (65\%) as uninsured. Overall, insured patients were older, more likely to be male, more likely to be white, and less likely to be Hispanic compared to uninsured patients (all $\mathrm{p}<0.05$ ). Additionally, insured patients were more likely to be men who have sex with men, and more likely to be HIVinfected compared to uninsured patients (all $p<0.0001$ ). Among asymptomatic patient visits, insured patients were less likely to have a diagnosis of chlamydia at any site or a diagnosis of rectal gonorrhoea.

In our municipal STD clinic, over one-third of patients currently report having insurance, yet still choose to seek care at the STD clinic. These data suggest that the expansion of access to insurance may not result in a reduced need for categorical STD services. Confidentiality and cost may be reasons for continued use of STD clinics among the insured. Maintaining access to high quality sexual health services should remain a priority in the era of expanded health care access.

\section{P3.059 EFFECT OF VAGINAL WASHING ON LACTOBACILLUS COLONISATION IN HIV-NEGATIVE KENYAN WOMEN}

doi:10.1136/sextrans-2013-051184.0519

1.2J E Balkus, ${ }^{3} \mathrm{G}$ Manguro, ${ }^{3} \mathrm{~A}$ Abdalla, ${ }^{3} \mathrm{C}$ Ngacha, ${ }^{3} \mathrm{~J}$ Shafi, ${ }^{3} \mathrm{~J}$ Kiarie, ${ }^{3} \mathrm{~W}$ Jaoko, ${ }^{2,3} \mathrm{R}$ S McClelland. 'Fred Hutchinson Cancer Research Center, Seattle, WA, United States; 2University of Washington, Seattle, WA, United States; ${ }^{3}$ University of Nairobi, Nairobi, Kenya

Background Vaginal washing has been associated with an increased risk of bacterial vaginosis (BV) and a decreased likelihood of vaginal Lactobacillus colonisation. We sought to determine whether a lower prevalence of Lactobacillus colonisation in women reporting vaginal washing was independent of the effect of BV.

Methods We conducted a cross-sectional study of 273 HIV-negative female sex workers enrolled in an open cohort study in Mombasa, Kenya. Vaginal washing and sexual risk behaviours were assessed using structured face-to-face interviews. Lactobacillus species were detected by plating vaginal swabs on both Rogosa and Columbia 5\% sheep blood agars. We used tetramethylbenzidine agar subculture to assess $\mathrm{H}_{2} \mathrm{O}_{2}$-production. BV was detected by Gram stain. Log-binomial regression was used to assess correlates of Lactobacillus colonisation, including vaginal washing, controlling for BV.

Results Two-hundred eighteen participants (80\%) reported vaginal washing in the past week (median frequency per week $=14$; range 1-35). Lactobacillus species were detected in 50/218 (23\%) participants who reported vaginal washing versus $23 / 55$ (42\%) who did not report this practise. Similarly, $\mathrm{H}_{2} \mathrm{O}_{2}$-producing Lactobacillus species were detected in $13 / 218(6 \%)$ participants who reported vaginal washing versus $10 / 55$ (18\%) who did not. After controlling for age, unprotected sex, and $\mathrm{BV}$, vaginal washing was associated with a lower likelihood of any Lactobacillus (adjusted relative risk $[a R R]=0.55 ; 95 \%$ confidence interval $[\mathrm{CI}] 0.37-0.81)$ and $\mathrm{H}_{2} \mathrm{O}_{2}$ producing Lactobacillus ( $\mathrm{aRR}=0.33$; 95\% CI 0.15-0.73).

Conclusion Vaginal washing was associated with a lower likelihood of any Lactobacillus and $\mathrm{H}_{2} \mathrm{O}_{2}$-producing Lactobacillus species detected by culture. The results of our adjusted analysis suggest that the effect of vaginal washing on lactobacilli is not mediated entirely through the higher prevalence of BV associated with this practise. Prospective studies will be important to determine whether cessation of vaginal washing could improve vaginal health by promoting vaginal colonisation with Lactobacillus.

\section{P3.060 THERE IS A NEED FOR MULTIPURPOSE PREVENTION TECHNOLOGIES TARGETING HIV AND COMMON REPRODUCTIVE TRACT INFECTIONS: DATA FROM THE MICROBICIDE SAFETY BIOMARKERS STUDY TEAM}

doi:10.1136/sextrans-2013-051184.0520 
${ }^{1}$ M Mwaura, ${ }^{2} \mathrm{~L}$ Hardy, ${ }^{3} \mathrm{~S}$ Delany-Moretlwe, ${ }^{4} \mathrm{G}$ Ndayisaba, ${ }^{1} \mathrm{~K}$ Mandaliya, ${ }^{5,6} \mathrm{R}$ Verhelst, ${ }^{5} \mathrm{H}$ Verstraelen, ${ }^{4,7} \mathrm{~J}$ van de Wijgert, ${ }^{2} \mathrm{~T}$ Crucitti, ${ }^{2} \mathrm{~V}$ Jespers. ${ }^{1}$ International Centre for Reproductive Health, Mombasa, Kenya; 'Institute of Tropical Medicine, Antwerp, Belgium; 'Wits Reproductive Health and HIV Institute, Johannesburg, South Africa; ${ }^{4}$ Rinda Ubuzima, Kigali, Rwanda; ${ }^{5}$ University of Ghent, Ghent, Belgium; ${ }^{6}$ International Centre for Reproductive Health, Ghent, Belgium; 'University of Liverpool, Liverpool, UK

Background The ideal vaginal microbicide should reduce the risk of HIV infection and other reproductive tract infections (RTIs) while preserving the integrity of the cervicovaginal epithelium. Future microbicides and multipurpose prevention technologies (MPT) could improve maternal reproductive health and prevent multiple sexually transmitted infections.

Objectives and Methods The Microbicide Safety Biomarkers Study is a prospective cohort study of 110 adults, 30 adolescents and 30 pregnant women in Kenya and South-Africa, 30 women engaging in vaginal practises in South-Africa and 30 high-risk and 30 HIV-positive women in Rwanda. RTIs and biomarkers of the vaginal microbiome and inflammation were studied.

Results Baseline prevalence RTI data are presented in the table. A significant difference ( $p=0.027$ to 0.001 ) between the study groups was present for all RTIs except for Trichomonas vaginalis (TV). Neisseria gonorrhoeae (NG), syphilis and HSV-2 were associated $(p=<0.001$ ) with sexual risk taking behaviour (sex worker OR at least 3 partners last year OR at least one sexual partner with HIV in the past 3 months OR age first sex less than 15 years). HSV-2 was detected in $51.5 \%$ of the high risk-takers compared to $28.6 \%$ of the low risk-takers. For women with bacterial vaginosis (Nugent 7-10) Chlamydia trachomatis (CT) $(\mathrm{p}=<0.028)$ was present in $14.9 \%$ and TV $(p=<0.001)$ in $9 \%$ compared to $6.3 \%$ and $1.5 \%$ in women without BV (Nugent $0-3$ ), respectively.

\section{Abstract P3.060 Table 1}

\begin{tabular}{lllllll}
\hline Group & HSV-2 & CT & NG & Syphilis & TV & Candida \\
\hline Adults & $34.0 \%$ & $10 \%$ & $0.9 \%$ & $0 \%$ & $3.7 \%$ & $19 \%$ \\
Pregnant & $26.7 \%$ & $10 \%$ & $0 \%$ & $1.7 \%$ & $6.8 \%$ & $40 \%$ \\
Adolescents & $6.7 \%$ & $6.7 \%$ & $0 \%$ & $0 \%$ & $6.8 \%$ & $20 \%$ \\
Vaginal Practices & $45.2 \%$ & $26.7 \%$ & $3.3 \%$ & $0 \%$ & $14.3 \%$ & $33.3 \%$ \\
High risk & $46.6 \%$ & $10 \%$ & $6.7 \%$ & $6.7 \%$ & $10 \%$ & $10 \%$ \\
HIV-positive & $82.8 \%$ & $0 \%$ & $13.3 \%$ & $20 \%$ & $10 \%$ & $13.3 \%$ \\
\hline
\end{tabular}

Conclusion RTIs are common among African women targeted for microbicide trials. The introduction of a MPT targeting a combined prevention of HIV and HSV-2 is warranted in these populations.

\section{P3.061 MYCOPLASMA GENITALIUM DNA DETECTED FROM ADOLESCENT MALES IN A LONGITUDINAL COHORT}

doi:10.1136/sextrans-2013-051184.0521

'J A Williams, 'B Weaver, ${ }^{1}{ }^{2}$ B Van Der Pol, 'D Mi, ${ }^{1} \mathrm{~J}$ D Fortenberry. 'Indiana University School of Medicine, Indianapolis, IN, United States, 'Indiana University School of Public Health, Bloomington, IN, United States

Background Mycoplasma genitalium (MG) causes non-gonococcal urethritis as well as asymptomatic infections although most data on the incidence and natural history of MG is from adults.

Methods Participants were 14-17 year old men in a longitudinal study of STI and the urethral microbiome. Urine samples were collected monthly and batch tested retrospectively for MG DNA using PCR. Urine samples were tested in real-time for chlamydia, gonorrhoea, trichomonas and white blood cells (WBC): infections by these organisms were treated. White blood cell count (WBC) was measured by automated cell count of fresh urine. Dysuria and urethral discharge were self-reported on cell phone diaries.
Results Among 75 participants (mean age 16.0 at enrollment), 6 $(8.0 \%)$ men have at least one MG positive sample, with a total of 14 MG positive monthly urine samples. The prevalence of Chlamydia or gonorrhoea infection was 19/75 (25.3\%) and 1/75 (1.3\%), respectively. All but one participant was positive for at least two consecutive months, and one participant was positive for 4 consecutive months. One participant was positive only once, was co-infected with chlamydia, but treatment could not be confirmed. No other MG positive visits occurred simultaneously with other STI. None of the participants reported symptoms or sexual behaviours within a 15 day window of the positive visit. Average urine WBC was 21.8 $\mathrm{WBC} / \mathrm{ml}$ urine although only $3 / 14 \mathrm{MG}$ positive samples were associated with urine WBC $>28.5 / \mathrm{ml}$ (commonly used as a diagnostic threshold for pyuria).

Conclusions MG in adolescent men is more common than gonorrhoea, persistent without treatment for up to 120 days, and is typically not associated with symptoms or pyuria. These data add to emerging understanding of the prevalence and natural history of sexually transmitted MG and support the importance of more detailed understanding of sexual and reproductive health morbidity associated with these infections.

\section{P3.062 MYCOPLASMA GENITALIUM PREVALENCE AND RISK FACTORS AMONG YOUNG SEXUALLY ACTIVE WOMEN IN THE GENERAL POPULATION AND ATTENDING SEXUALLY TRANSMITTED INFECTION CLINICS IN LONDON, UK}

doi:10.1136/sextrans-2013-051184.0522

'S Dave, 'H Svenstrup, ${ }^{2} \mathrm{C}$ Carder, ${ }^{2 P}$ Grant, ${ }^{2} \mathrm{~S}$ Morris-Jones, 'I Kidd, ' $\mathrm{J}$ Stephenson. ${ }^{1}$ University College London, London, UK; ${ }^{2}$ University College London Hospitals NHS Foundation Trust, London, UK

Background Mycoplasma genitalium is a sexually transmitted infection (STI) associated with cervicitis, endometritis and pelvic inflammatory disease in women. There is a lack of data on M.genitalium in the United Kingdom. We conducted a study to determine its prevalence and risk factors among sexually active young women in the general population and attending STI clinics.

Methods First catch urine samples, self-taken vaginal and cervical swabs from 4644 women in the National Chlamydia Screening Programme (NCSP) and attending two London STI clinics were tested for $M$. genitalium by quantitative real-time PCR, confirmed by $\mathrm{MgPa}$ 1-3 genotyping. C.trachomatis results, demographic, sexual behaviour (NCSP only, 2470 women) and STI (clinics only, 2174 women) data were also available.

Results $M$. genitalium prevalence was $3 \%$, C.trachomatis $5 \%$ and only $0.5 \%$ of women were co-infected. M.genitalium was more prevalent in swab than urine samples (4.6\% vs. $1.4 \%, \mathrm{p}<0.001)$ with a significantly higher mean bacterial load. Among NCSP participants M.genitalium was associated with ethnicity (black $4.7 \%$ vs. white $2 \%, \mathrm{p}=0.01)$ and C.trachomatis with age (16-19 years $8.5 \%$ vs. $20-24$ years $5.7 \%, \mathrm{p}<0.01)$. M.genitalium and C.trachomatis were detected more frequently in women reporting multiple sexual partners in the previous year compared to women who reported only one partner (OR 2.2, p $=0.02$ and OR 1.8, $p<0.01$, respectively). Among STI clinic attendees $M$.genitalium was associated with younger age (1619 years $9.9 \%, 20-24$ years $6.2 \%$ vs. $>25$ years $1.7 \%, p=<0.01)$. Chlamydia prevalence was 6\% in STI clinic attendees aged 16-24. Women previously diagnosed with chlamydia or Trichomonas vaginalis were significantly more likely to have M.genitalium compared to women with no previous STI diagnoses (OR 2.4, $\mathrm{p}=0.02$ and OR 5.7, $\mathrm{p}<0.01$, respectively).

Conclusion $M$. genitalium and $C$. trachomatis seldom co-exist and appear to have different risk factors. Further data on M.genitalium are necessary to determine the need for routine testing and treatment. 